\title{
Genomics of the thermo-acidophilic red alga Galdieria sulphuraria
}

\author{
Guillaume G. Barbier, Marc Zimmermann, Andreas P.M. Weber* \\ Michigan State University, Department of Plant Biology, East Lansing, MI, USA, 48824-1312
}

\begin{abstract}
Extremophilic organisms dwell in environments that are characterized by high or low temperatures (thermophiles or psychrophiles), very low or high $\mathrm{pH}$-values (acidophiles or alkalophiles), high salt concentrations (halophiles), high pressure (barophiles), or extreme drought (xerophiles). Many extremophiles are microbes, and many also belong to the prokaryota. Galdieria sulphuraria, however, is a member of a group of extremophilic eukaryotes that are named Cyanidiales. Cyanidiales are unicellular red micro-algae that occur worldwide in hot acidic waters, volcanic calderas, and in human-made acidic environments such as acidic mine drainage. G. sulphuraria has a unique position within the Cyanidiales because, in contrast to the other obligate photoautotrophic members of this group, it is able to grow photoautotrophically, mixotrophically, and heterotrophically. It is not only resistant to acid ( $\mathrm{pH} 0$ ) and heat $\left(56^{\circ} \mathrm{C}\right)$, but also to high salt $(1.5 \mathrm{M} \mathrm{NaCl})$, toxic metals, and many other abiotic stressors. This unusual combination of features such as thermophily, acidophily, resistance to a wide array of abiotic stressors, and an extraordinary metabolic plasticity make G. sulphuraria highly interesting model organism to study adaptation to extreme environments. We have started a genomics approach to gain insight into the biology of G. sulphuraria and to identify genes and gene products critical for survival under extreme conditions. To this end, we pursue a wholegenome, shotgun sequencing approach towards unraveling the genome sequence of $G$. sulphuraria. We report here on the status quo of the genome-sequencing project and we summarize what we have learned to date from the genome sequence about the biology of this truly unique extremophile.
\end{abstract}

Keywords: Extremophilic, thermophilic, acidophilic, photosynthesis, red alga, genome sequencing, Cyanidiales, metabolic plasticity

\section{INTRODUCTION}

\subsection{Systematics and phylogenetic position of G. sulphuraria}

G. sulphuraria belongs to a small group of evolutionary anciently diverged, unicellular micro-algae called Cyanidiales. Based on molecular clock estimates, Cyanidiales are probably more than 1.5 billion years old and thus likely some of oldest extant eukaryotic organisms. Cyanidiales form an ancient monophyletic group within the red algae that is located at the root of secondary endosymbiosis ${ }^{1,2}$. These findings are supported by detailed studies of the evolution of starch metabolism in apicomplexa, rhodophytes, and chlorophytes ${ }^{3}$. Cyanidiales exist in many parts of the world in hot acidic habitats, both natural and human-made. This group consists of the three genera Cyanidioschyzon, Cyanidium, and Galdieria. Based on detailed morphological studies and the mode of cell division, six independent species have been established: Cyanidioschyzon merolae, Cyanidium caldarium, Galdieria maxima, Galdieria partita, Galdieria daedala, and Galdieria sulphuraria ${ }^{4}$. The taxonomic positions of Cyanidium, Cyanidioschyzon and Galdieria have been recently updated ${ }^{5}$ and the proposed taxonomy is in concordance with recent molecular evidence ${ }^{6,7}$. In this manuscript, we will address Galdieria sulphuraria (Galdieri) Merola ${ }^{6}$, the metabolically most versatile member of the Cyanidiales. It is important to note that much of the classic literature on Cyanidium caldarium actually applies to Galdieria sulphuraria, due to previous uncertainties in the phylogenetic positions of both organisms.

* aweber@msu.edu; phone 1517 432-1436; fax 1517 432-5294; http://plantbiology.msu.edu/weber.shtml

Astrobiology and Planetary Missions, edited by Richard B. Hoover, Gilbert V. Levin, Alexei Y. Rozanov, G. Randall Gladstone, Proc. of SPIE Vol. 5906, 590609, (2005) - 0277-786X/05/\$15 - doi: 10.1117/12.614532 


\subsection{Cyanidiales are the only photosynthetic organisms able to thrive in hot acid}

Most extremophiles are microorganisms. For example, the presently known upper temperature limits are $113^{\circ} \mathrm{C}$ for archaeal, $95^{\circ} \mathrm{C}$ for bacterial, and $62^{\circ} \mathrm{C}$ for eukaryotic microorganisms. With few exceptions, metazoans are unable to grow above $50^{\circ} \mathrm{C}^{8,9}$.

Surprisingly, photosynthetic prokaryotes such as the cyanobacteria are completely absent from hot acid waters ${ }^{10}$. Instead, thermo-acidophilic photosynthetic unicellular red algae of the Cyanidiales are the principal photosynthetic organisms found in these ecological niches ${ }^{8,11,12}$. These obligate acidophilic eukaryotic microorganisms can grow at $\mathrm{pH} 0$ and temperatures up to $56^{\circ} \mathrm{C}^{13,14}$.

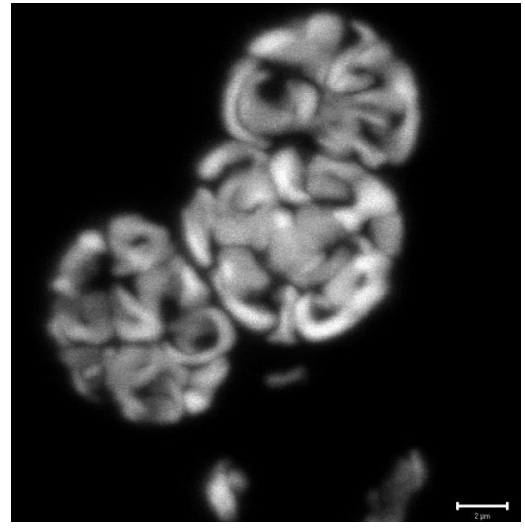

Figure 1: Confocal laser scanning image of three $G$. sulphuraria cells. Excitation wavelength was $543 \mathrm{~nm}$ and a $650 \mathrm{~nm}$ long pass emission filter was used. The image shows chlorophyll autofluorescence of the tubular shaped chloroplasts in each cell. The scale bar to the lower right is $2 \mu \mathrm{m}$.

\subsection{Galdieria sulphuraria is an extraordinarily stress tolerant and} metabolically versatile microorganism

G. sulphuraria naturally inhabits volcanic environments, such as hot sulfur springs and solfatara soils ( $\mathrm{pH} 0$ to 4 and temperatures up to $56^{\circ} \mathrm{C}$ ), but also equally hostile environments originating from human activities such as strip or opencast mining. Galdieria frequently represents up to $90 \%$ of the total biomass and almost $100 \%$ of the eukaryotic biomass under these conditions. Cyanidiales are the principle photosynthetic organisms in these ecological

niches.

Galdieria sulphuraria is able to grow photoautotrophically, mixotrophically, and heterotrophically ${ }^{15}$. It thrives on more than 50 different carbon sources such as sugars, sugar alcohols and amino acids ${ }^{15-18}$, a metabolic flexibility matched by few other organisms.

In addition, G. sulphuraria tolerates up to $9 \%(\mathrm{w} / \mathrm{v})$ salinity ${ }^{5}$ and its cells possess a very rigid protein-rich cell wall ${ }^{19,20}$. Galdieria thrives in pure $\mathrm{CO}_{2}{ }^{21}$ and elevated atmospheric pressure, leading to the proposal that it could survive even on Venus ${ }^{22}$.

Acidic environments are frequently characterized by high concentrations of iron, cadmium, aluminum, nickel, and other metals that are highly toxic to almost all organisms. G. sulphuraria is resistant to high levels of toxic metal ions including $200 \mathrm{mM}$ aluminum. The resistance is likely due to active secretion of metal ions ${ }^{23-25}$ but such a mechanism has yet to be confirmed.

Surprisingly, G. sulphuraria, in contrast to acidophilic green algae such as Dunaliella acidophila, is able to maintain a proton gradient of 1:1 million across its plasma membrane during several months of continuous darkness ${ }^{26}$, indicating an energy independent proton-exclusion mechanism. The mechanism by which this temporary impermeability to protons is achieved is also unclear. It has been hypothesized, based on studies with archaebacteria, that the incorporation of sterols, saturated fatty acids, bipolar tetraether lipids, and proteins could account for this impermeability ${ }^{27-30}$. Very long chain dicarboxylic acids are thought to have similar roles in some eubacteria ${ }^{31-33}$. Possibly also the rigid, protein-rich cell wall is involved in excluding protons.

\subsection{Life in hot acid and its relation to protein structure}

Photosynthesis in hot, acidic environments is challenging because of an increased oxygenation reaction of ribulose 1,5-bisphosphate carboxylase/oxygenase (RubisCO) at high temperature, and a strong decrease of dissolved inorganic carbon availability at low $\mathrm{pH}$. Therefore, specific adaptations of the carbon assimilatory apparatus to these extreme conditions are to be expected. Galdieria RubisCO is relatively heat stable ${ }^{34}$. The crystal structure of RubisCO from G. partita was recently solved and it was demonstrated that this enzyme had the highest specificity in the direction of the carboxylation reaction of RubisCOs hitherto reported ${ }^{35}$. Galdieria Rubisco has a unique hydrogen bond between the main chain oxygen of Val332 on loop 6 and the epsilon-amino group of Gln386 of the same large subunit. It was proposed that this interaction is crucial for stabilizing loop 6 in the closed state and for generating a higher affinity for anionic ligands ${ }^{36}$. The enhanced thermo-stability of Galdieria proteins ${ }^{34,37-39}$ should facilitate efforts to gain structural information. We expect Galdieria proteins to become an important resource for structural studies of soluble and membrane proteins; in particular those, that do not have orthologs in prokaryotic extremophiles. Functional genomics of G. sulphuraria and other eukaryotic extremophiles will open up the way to 
explore the molecular mechanisms of eukaryotic live under extreme conditions. In addition, it will provide a rich source of genes with high potential for bioengineering, novel metabolites, stress resistance, and bioremediation.

\subsection{Comparative genomics of Cyanidiales}

Comparative genomics (i.e., the comparison of genomes of different but related organisms) is a powerful tool to unravel the molecular foundations of observable traits and phenotypes that cannot easily be deduced from the analysis of individual genome sequences. Its core hypothesis is that conserved regions of DNA between two species often encode for the common features of the organisms, while different traits will appear as differences in the genetic makeup between the two species ${ }^{40}$. Similar genomes, such as those of Galdieria and Cyanidioschyzon, are particularly useful for elucidating key differences that account for structural and metabolic differences in these organisms. Using the recently published genome sequence of C. merolae ${ }^{41}$ and a G. sulphuraria genomic dataset consisting of an EST-collection ${ }^{42}$ and $8 \mathrm{Mbp}$ of non-redundant genomic sequence (approximately 40-50\% genome coverage; http://genomics.msu.edu/galdieria), we have recently conducted a comparative genomics pilot study to identify those genes of $G$. sulphuraria that might be crucial to its extraordinary metabolic flexibility ${ }^{43}$. To this end, we identified Galdieria sequences that did not match any of the C. merolae genes and that displayed similarity to genes of known function from other organisms. Although only $50 \%$ of the G. sulphuraria genome sequence had been finished at the time of the study, a number of important conclusions could be drawn: (i) Despite their evolutionary distance, the Cyanidiales have retained a high level of overall-similarity in their genomes, (ii) Galdieria genes contain more introns than those of Cyanidioschyzon, (iii) only Galdieria is capable of metabolizing complex cell wall polysaccharides, (iv) the lack of heterotrophy in Cyanidioschyzon is not accompanied by a strong reduction in its carbohydrate metabolism enzymatic make-up, and (v) the Galdieria genome encodes many more membrane transporters than that of Cyanidioschyzon ${ }^{43}$.

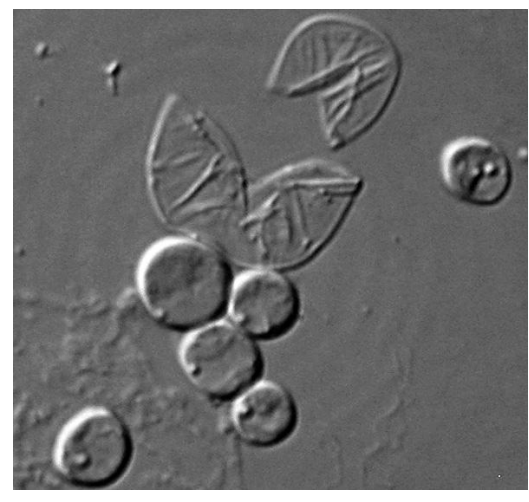

Figure 2: Light micrographic image showing intact G. sulphuraria cells and two empty cell walls. G. sulphuraria divides by endospores. After two mitotic divisions, the four daughter cells (endospores) rupture the cell wall of the mother cell and are released to the environment. The empty cell wall of the mother cell remains.

This pilot study was based on genomic fragments and EST sequences. Complete and reliable gene models for G. sulphuraria will significantly increase the value of its genome sequence for comparative genomic approaches and we expect widespread use of these data in comparative studies.

\section{METHODOLOGY}

\subsection{Isolation of genomic DNA from G. sulphuraria}

G. sulphuraria cells were harvested from heterotrophic cultures ${ }^{16}$ by centrifugation, frozen in liquid nitrogen, and ground to a fine powder by mortar and pestle. Total nucleic acids were extracted by incubating the ground tissue overnight in $50 \mathrm{mM}$ Tris-Cl pH7.5, $5 \mathrm{mM}$ EDTA, and 1\% (w/v) SDS, followed by extraction of proteins with phenol:chloroform:isoamylalcohol (24:24:1), and precipitation of DNA from the aqueous phase by ethanol. The pellet was dissolved in $10 \mathrm{mM}$ Tris- $\mathrm{Cl} \mathrm{pH}$ 7.5, $1 \mathrm{mM}$ EDTA, and RNA was removed by incubation with DNAsefree RNAse, followed by de-proteination with phenol:chloroform:isoamylalcohol, and DNA-precipitation by ethanol. Genomic DNA was further purified by CsCl-density gradient centrifugation of bis-benzamide-treated total DNA as described previously ${ }^{44}$.

\section{$2.2 \quad$ Construction of genomic DNA libraries}

Small insert plasmid libraries: Small-insert shotgun sequencing plasmid libraries were constructed in pSMART-HC Kan (Lucigen Corp., Middleton, WI; www.lucigene.com). Genomic DNA was randomly fragmented by shearing using a HydroShear device ${ }^{45}$ and end repaired to generate blunt ends. Fragments of approximately $2 \mathrm{kbp}$ (isolated 
by preparative agarose gel electrophoresis) were ligated into the pSMART vector and plasmids were transformed into E.cloni cells (Lucigen).

Fosmid library: A fosmid library containing $40 \mathrm{kbp}$ inserts was constructed by physically shearing genomic DNA and ligation of end repaired, size fractionated DNA into the Fosmid vector pCC1FOS, followed by packing into Lambda phages.

BAC library: Two BAC libraries (BamHI, EcoRI) containing $100 \mathrm{kbp}$ inserts were constructed using the CopyControl BAC Cloning Kit (Epicentre Technologies, Madison, WI) according to instructions by the manufacturer. High molecular weight genomic DNA embedded in LMP agarose plugs was partially digested by EcoRI or BamHI, respectively. Fragments were size fractionated by pulsed-field gel electrophoresis and isolated by electro-elution. Fragments were ligated into predigested and dephosphorylated pCCBAC vectors.

\subsection{Plasmid isolation and sequencing}

A GeneMachines Mantis colony picker was used to pick bacterial colonies into 96-well plates. Qiagen 3000 robots were used for liquid handling and plasmid purification, in addition to a GeneMachines RevPrep Orbit plasmid purification system. Sequencing reactions were performed on a PE9700 thermocycler and an ABI 3730x1 highthroughput capillary DNA sequencing systems was used to acquire sequence information. All sequence data and chromatograms were stored on a Geospiza Finch server (Geospiza, Seattle, WA).

\section{$2.4 \quad$ Sequence data and similarity searches}

Cyanidioschyzon merolae predicted peptide sequences were obtained from http://merolae.biol.s.u-tokyo.ac.jp (Matsuzaki et al., 2004), data released March 16, 2004. Genomic sequences of G. sulphuraria were screened against a database consisting of Porphyra purpurea chloroplast, Cyanidium caldarium str. RK1 chloroplast, and Cyanidioschyzon merolae plastid and mitochondrion sequences as published in Genbank. EST libraries and sequences were reported previously ${ }^{42}$. Similarity searching was done with the BLAST 2.2.6 program (NCBI) ${ }^{46}$. Data were parsed and analyzed using software implementations developed by the Genomic Technology Support Facility (GTSF) at Michigan State University (http://genomics.msu.edu).

\section{RESULTS AND DISCUSSION}

\subsection{Current status of the genome-sequencing program}

Literature data on the genome size of Galdieria spec. vary between $10.8 \mathrm{Mb}$ and $14.2 \mathrm{Mb}$ : Using microspectrophotometry, a genome size of $10.8 \mathrm{Mbp}$ was estimated for G. sulphuraria $074 \mathrm{~W}^{47}$. Genome sizes ranging between 9.8 Mbp (G. sulphuraria 19.71) and 14.2 Mbp (Galdieria spec. isolates, Rio Tinto, Spain) were determined using pulsed-field gel electrophoresis ${ }^{48}$. The genome of the related species $C$. merolae was recently finished and its 16.5 Mb encode 5,331 genes ${ }^{41}$. At the time of writing (June 2005), we have obtained 153,814 random sequence reads from small-insert plasmid libraries, and from cosmid and BAC ends. These equal 94.3 Mb of Q20 bases and 15.5 Mb of non-redundant sequence (5.1-fold coverage), indicating that the Galdieria genome is larger than expected from literature data. Based on the scaffold derived from BAC and cosmid end sequencing, we estimate a final genome size between 18 and $20 \mathrm{Mb}$, similar to the genome of $C$. merolae. Our preliminary results (pulsed-field gels; genome sequence contigs) indicate that $G$. sulphuraria has several very small chromosomes $(<200 \mathrm{kB})$ and we believe this is one of the reasons why the genome size was underestimated previously. Monthly updates on the sequencing statistics can be found at: http://galdi.bch.msu.edu/cgi-bin/galdieria/galdi2.pl.

\subsection{Results from EST-sequencing and analysis}

Two directionally cloned cDNA libraries were generated from photoautotrophically and heterotrophically grown $G$. sulphuraria cells, respectively, and 5,915 cDNA inserts were sequenced from their 5'-ends. 3,323 represented the autotrophic library, and 2,592 represented the heterotrophic library. Base calls were screened for vector and $E$. coli contamination, and quality values were assigned to each base using the phred algorithm ${ }^{49-51}$. 5,270 sequence reads (89\% of the total) passed the filter, and the final dataset consisted of 3,024 and 2,246 sequences generated from the autotrophic and heterotrophic libraries, respectively. The average phred Q20 ${ }^{49}$ read length was 563 bases. The 5,270 passing sequences were clustered using the stackPACK software package (South African National Bioinformatics 
Institute, University of the Western Cape, Republic of South Africa), resulting in 3,047 contigs (1.7 Mbp) of unique, non-redundant sequence.

Of these 3,047 contigs, 1,746 and 1,276 consisted of ESTs from only the autotrophic or heterotrophic libraries, respectively. Little overlap was found between both libraries, only 34 contigs contained sequence information derived from both libraries. This is most likely due to: (i) significantly different gene expression levels under both conditions, hence each library represents a snapshot of the expression profile under a specific culture condition rather than a comprehensive collection of cDNA clones. (ii) a relatively high "novelty rate" that is usually obtained during the early phases of random sequencing projects such as EST sequencing ${ }^{52} .2,431$ ESTs (80\%) were unique sequences, whereas only $20 \%$ of the 3,047 contigs contained more than one EST sequence. This indicates that we did not reach saturation; hence only little overlap between both libraries is to be expected. For initial analysis of EST-sequences, we placed emphasis on the reconstruction of metabolic pathways. We found evidence for (i) a complete pathway for lipid A biosynthesis; (ii) export of triose-phosphates from rhodoplasts; (iii) and absence of eukaryotic hexokinases. A detailed description of the results was recently published ${ }^{42}$.

\subsection{Intron structure}

A comparison of the G. sulphuraria genomic contigs to the EST dataset showed that more than $50 \%$ of all tested Galdieria genes contain introns. Based on 10 randomly selected genes containing one to three introns, intron lengths of 45 to 65 bases were found ${ }^{43}$. The borders of the introns displayed typical spliceosomal features as previously described for a LHC gene from G. sulphuraria ${ }^{53}$ : in $80 \%$ of the cases, the splice donor site started with the sequence GU and the same percentage was observed for the splice acceptor site, which consisted of AG. In only $50 \%$ of the cases the branching site was composed of the consensus sequence CUPuAPy located 15 to 30 bases upstream of the acceptor site.

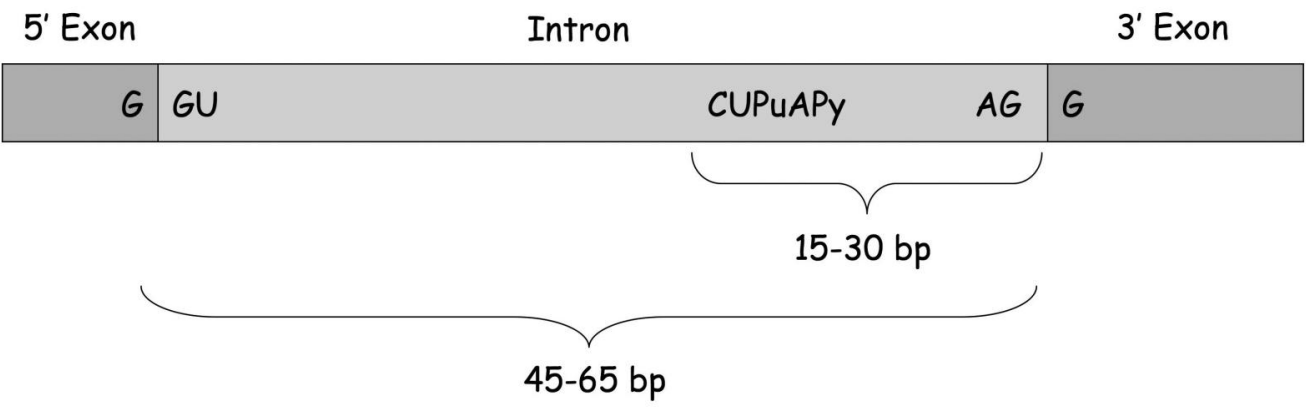

Figure 3: Typical intron structure in G. sulphuraria. The splice donor and acceptor sites and the consensus branching site are indicated.

\subsection{Current bioinformatics tools do not correctly predict gene models in G. sulphuraria}

The Galdieria genome has several unusual features, such as very small introns (45 to $60 \mathrm{bp}$ ) and short intergenic regions. These specific features of the Galdieria genome complicate ab initio gene predictions using programs such as GenScan ${ }^{54}$ or GeneMark.hmm ${ }^{55}$. In a pilot study using 10 experimentally verified full-length cDNA sequences, we found that none of the tested programs (GenScan, GlimmerR, FGENESH, GeneMark.hmm) precisely predicted more than $60 \%$ of gene structures. The best results (60\% correct models) were obtained using GeneMark.hmm (trained on Arabidopsis, which has a relatively similar $\mathrm{G}+\mathrm{C}$ contents). In particular the small introns $(<100 \mathrm{bp})$ proved to be problematic and frequently adjacent genes were sliced together, leading to gene fusions. Although the accuracy of gene predictions can be improved by combining several programs ${ }^{56}$, or by training predictors with genomes showing similar characteristics ${ }^{57}$, it becomes clear that reliable gene models require experimental support from cDNAs. In addition, cDNA sequences are required to build training sets for $a b$ initio gene predictors to improve the quality of the computational predictions.

\subsection{Mining the G. sulphuraria genome for membrane proteins}

G. sulphuraria represents a particular interesting species for structural genomics and proteomics projects owing to its extraordinary metabolic versatility such as heterotrophic and mixotrophic growth on more than 50 different carbon sources. Moreover, it also tolerates high concentrations of toxic metals such as cadmium, mercury, aluminum 
or nickel. Hence, the genome of G. sulphuraria may provide membrane protein targets that are particularly interesting from a biotechnology standpoint, because of their thermostability.

Eukaryotic cells are highly compartmentalized, allowing the parallel occurrence and efficient spatial separation of metabolic pathways that are competing for mutual precursors and metabolic intermediates. Examples are the tricarboxylic acid cycle in mitochondrial matrix, the glycosylation and sulphatation of proteins in the endomembrane system, and the Calvin cycle in the chloroplasts stroma of plant cells. Many of the products that are synthesized in one compartment are further metabolized in other cellular compartments. Therefore, the functioning of eukaryotic cells strongly relies on a complex metabolic network that intertwines reactions in between intracellular compartments by the exchange of metabolic intermediates. The organellar membranes represent the interface between cellular compartments and contain numerous transport proteins that mediate the flux of metabolites and ions across these membrane(s).

Many intracellular transporters do not have related proteins in prokaryotic organisms. In particular, solute transporters of the mitochondrial membrane, the Golgi/ER-endomembrane system, and of the plastid inner envelope membrane are confined to eukaryotic organisms. In contrast to intracellular transport processes that are exclusive to eukaryotic cells, the transport of solutes across the plasma membrane is crucial for both eukaryotic and prokaryotic organisms and many of the corresponding transport systems can be identified in both kingdoms.

The ongoing genome project for G. sulphuraria (http://genomics.msu.edu/galdieria) and the completely sequenced genome of C. merolae (http://merolae.biol.s.u-tokyo.ac.jp/) offer for the first time unique possibilities for a global analysis of the in silicio predicted proteomes of thermophilic eukaryotes as a whole or as a subset of proteins, e.g. membrane proteins. Transmembrane proteins contain in general from one to over 14 transmembrane spans in the form of $\alpha$-helices. There are also $\beta$-barrel proteins with an even number of $\beta$-strands found in the outer membrane of bacteria, mitochondria, and plastids.

The predicted number of transmembrane proteins present in the genome of $C$. merolae amounts to approximately 1,250 proteins containing one ore more transmembrane spans, corresponding to $22 \%$ of the 6,390 proteins encoded by its genome ${ }^{41}$. Based on a preliminary genome annotation for $G$. sulphuraria, the genome of this alga encodes a higher portion of membrane proteins, as is to be expected because of its ability to take up external carbon sources ${ }^{43}$. Many membrane proteins from Cyanidiales can be classified by the transporter classification (TC) system, a functional/phylogenetic system designed for the classification of all transmembrane transport proteins ${ }^{58,59}$ down to the transporter family level (third digit of the TC system). With few exceptions, at least one member of a particular TC-family can be identified in either G. sulphuraria or C. merolae. In an initial survey, 122 C. merolae membrane proteins and 133 G. sulphuraria membrane proteins have been classified by the TC system (data not shown), clearly demonstrating that members of many TC-families are represented in thermophilic Cyanidiales genomes. These unicellular microalga hence represent a rich source of genes encoding potentially thermo-stable membrane transporters that are highly interesting targets for structural studies. Of particular interest are the many members of the mitochondrial transporter superfamily (57 genes), of the organic cation transporter family (16 genes), sugar porter family (25 genes), the ATP-binding cassette (ABC) transporter family (32 genes), and the drug-metabolite transporter (DMT) family (17 genes).

Below we provide a brief summary of our initial survey of the Galdieria genome for membrane proteins that are highly homologous to mammalian and bacterial membrane proteins and that we consider interesting targets for functional and structural studies.

3.5.1 Membrane-bound glycosyltransferases. Many of the important glycosyltransferases involved in membrane biogenesis are integral membrane proteins or interfacial proteins. Several important families of glycosyltransferases catalyze the formation of monoglycosyl-sterols, monoglycosyl-ceramide, and glycosylated diacylglycerol, a variety of glycolipids and second messengers. Several genes of Galdieria encode proteins that are highly homologous to mammalian (dolichyl-phosphate mannosyltransferase and O-linked $\mathrm{N}$-acetylglucosamine transferase), plant (1,2diacylglycerol 3-beta-galactosyltransferase) and bacterial (lipid A disaccharide synthase) enzymes. This work has implications for the development of antibacterial and antifungal agents.

3.5.2 Transporters (active, symport, antiport, etc). We have identified many genes encoding solute transporters in Galdieria and several of these targets are being cloned for expression studies. One particular interesting target is 
the plastidic ADP/ATP translocator. It is unrelated to the mitochondrial ADP/ATP carrier, but exhibits significant homology to the plastid nucleotide translocators from higher plants and to the plastidic ADP/ATP translocator from Chlamydia, a major human and animal pathogen ${ }^{60-62}$.

In addition, we have identified several putative E1-E2 ATPases in Galdieria that are homologous to chromaffin granule ATPase II, which is involved in phospholipid transport. From the very large family of E1-E2 ATPases, only the structure of $\mathrm{Ca}^{2+}$-ATPase has been determined ${ }^{63,64}$ and there are still many unanswered questions about the structure and function of this critically important family of pumps.

3.5.3 Membrane Proteins involved in Organellar Biogenesis Within the genome of Galdieria, several genes have been identified that are homologous to mammalian genes whose products are involved in cell and organelle division. Genetic defects of cell and organelle division are quite deleterious and defects in mitochondrial division and fusion is associated with a diverse set of human diseases ${ }^{65}$. We have already identified a gene that is closely homologous to the mitochondrial fission protein Dlp1. The existence of the dynamin-related protein Dlp1/Dnm1 suggests that Galdieria abandoned a bacterial-like mitochondrial division process involving FtsZ ${ }^{66-68}$ shortly after the symbiotic fusion that gave rise to early eukaryotes like Galdieria. We have also identified another gene that is closely homologous to a mitochondrial FtsH-like AAA metalloprotease; the gene in Galdieria also displays surprisingly high sequence homology $\left(\mathrm{E}-\mathrm{value}=1 \mathrm{e}^{-101}\right)$ to the transitional endoplasmic reticulum ATPase from humans. The structural analysis of these proteins from humans, plants, and an early eukaryote like Galdieria would not only shed light on the evolution of organelle biogenesis in eukaryotes, but also provide a better understanding of the impact of organelle defects on cell physiology.

\subsubsection{Phosphate translocators and other members of the Drug/Metabolite Transporter (DMT) Superfamily -}

Plastidic phosphate translocators (PTs) form a sub-group of the DMT-family that cannot be assigned with the typical six or twelve helix topologies that are found in many other membrane transporter families. To date, only one structure of a member of the DMT-family (EmrE) has been solved at 3.8A resolution and this protein has four transmembrane domains ${ }^{69,70}$. The by far best-studied members of the DMT-family are the phosphate translocators of plant cell plastids. Similar to many other proteins of the DMT-family, these proteins have most likely 8 to 10 transmembrane domains ${ }^{71}$. Plant phosphate translocators are related to a large family of membrane proteins in plants, animals, and fungi ${ }^{72}$ that all belong to the DMT-family. The structure of plant phosphate translocators might thus be considered representative for a large number of intracellular transport proteins. In contrast to most other members of the DMT superfamily, pPTs are functionally characterized, and, importantly, heterologous expression in yeast, affinity purification of recombinant transporter protein to apparent homogeneity by metal-affinity chromatography, and functional assay of transporter activity after reconstitution into liposomes has been established for all members of this protein family ${ }^{73-78}$. Similar expression and reconstitution systems have been used for structurally and phylogenetically unrelated dicarboxylate transporters of the plastid envelope membrane ${ }^{79,80}$, indicating that this experimental strategy is potentially applicable to plastid envelope membrane proteins in general. The triose-phosphate/phosphate translocator is the dominant protein of the chloroplast membrane, representing up to $15 \%$ of the total membrane protein ${ }^{81}$, suggesting that high amounts of these transporter proteins can accumulate in the native membrane environment. A highly specific inhibitor for phosphate translocators is available that binds in a 1:1 stoichiometry to a lysyl-residue in the active site of native protein ${ }^{82-84}$, indicating this inhibitor could be used to rigidify the structure of the transport protein during crystallization trials.

It has been previously demonstrated that UDP-galactose-4-epimerase from G. sulphuraria is an unusually thermostable enzyme that was not inactivated even after incubation at $46^{\circ} \mathrm{C}$ for several hours ${ }^{38}$. It is tempting to hypothesize that this also applies to membrane proteins of these thermophilic organisms. A survey of the genome sequences of $G$, sulphuraria and $C$. merolae revealed the presence of small gene families encoding PT-related proteins in both thermophilic algae ${ }^{43}$.

In a preliminary study, we have tested whether the expression and reconstitution systems established for phosphate translocators from mesophilic plants can be applied to orthologous proteins from G. sulphuraria. The recombinant protein could be expressed at very high yields (not shown). In addition, the recombinant protein was active after reconstitution into liposomes, i.e., we were able to detect high rates of phosphate uptake into liposomes reconstituted with recombinant translocator protein. Figure 4 shows that recombinant, reconstituted phosphate translocator of the thermophilic algae G. sulphuraria 4 -fold higher activity at $40^{\circ} \mathrm{C}$ in comparison to $20^{\circ} \mathrm{C}$, indicating reduced conformational flexibility at room temperature and higher thermal stability of the transport protein, a desirable feature for structural studies. 


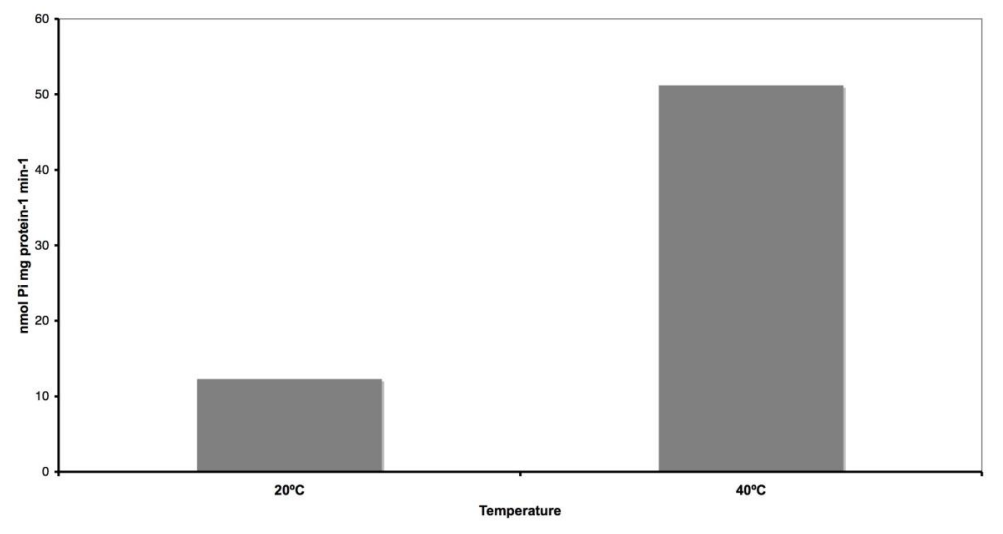

Figure 4: Temperature dependency of phosphate uptake into liposomes that had been reconstituted with a plastidic phosphate translocators from G. sulphuraria and preloaded with $20 \mathrm{mM}$ unlabeled orthophosphate. The rate of phosphate uptake was found to be 4 -fold higher at $40^{\circ} \mathrm{C}$ as compared to $20^{\circ} \mathrm{C}$.

\subsection{Pathway of carbon export from red algal chloroplasts}

Chloroplasts are the sites of photosynthetic carbon assimilation in plant cells. The net product of carbon assimilation by the reductive pentose phosphate pathway, triose phosphate, serves as principle precursor for all biosynthetic reactions in plants. In green plants, recently assimilated carbon is shuttled into starch and sucrose biosynthesis, nitrogen and sulfur metabolism, fatty acid biosynthesis, cell wall biosynthesis, secondary metabolism, and a puzzling variety of other metabolic routes. In red algae, the predominant soluble carbohydrate is floridoside ( $\alpha$-Dgalactopyranosyl-(1,2)-glycerol), not sucrose, and in contrast to green plants, red algae store starch in the cytosol, not inside the chloroplast. A key question in plant physiology is how the allocation of carbon to the individual pathways is regulated. The initial step in the allocation of recently assimilated carbon to different metabolic routes is the partitioning of triose phosphates between the plastid stroma and the plant cell cytosol. In addition, reduced carbon can be withdrawn from the regenerative phase of the Calvin Cycle, for example to fuel the plastid-localized shikimic acid pathway. In green plants, carbohydrates that are synthesized in chloroplasts by the reductive pentose phosphate pathway during the day are mainly exported to the cytosol as triose phosphate by the triose phosphate/phosphate translocator (TPT ${ }^{85-87}$ ). The predominant fate of triose phosphate in the cytosol is the conversion to sucrose, which serves as the transport form of photoassimilates that are allocated to sink tissues. We asked the question as to whether a similar pathway for the export of carbohydrates from chloroplasts exists in red algae. A survey of the G. sulphuraria and C. merolae genomes indicated that the genomes of both red algae harbor genes that encode proteins that show significant homologies to plastidic phosphate translocators from green plants ${ }^{42}$. Liposomes reconstituted with total G. sulphuraria membrane proteins showed the signature phosphate/phosphate antiport activity characteristic for plastidic phosphate translocators and we also could detect triosephosphate/phosphate counter-exchange ${ }^{42}$. We thus propose that the export of recently assimilated carbon from red algal chloroplasts occurs by a similar counter-exchange mechanism as in green plant chloroplasts and that a protein similar to plastidic triosephosphate/phosphate translocators from green plants catalyzes this counterexchange (see Figure 5).

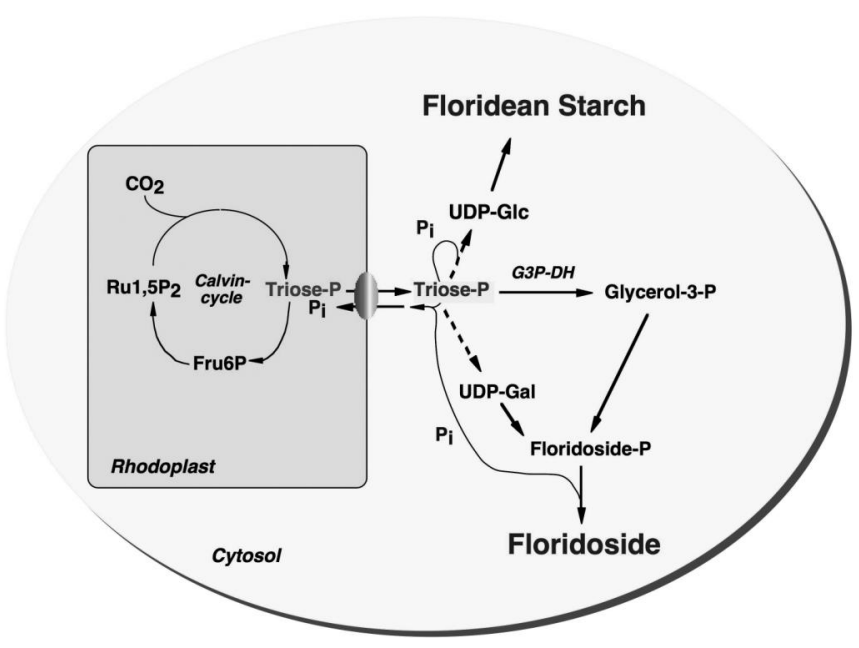

Figure 5: Proposed model for carbon export from red algal chloroplasts. $\mathrm{CO}_{2}$ is assimilated by the Calvin Cycle in the red algal chloroplast (Rhodoplast), yielding reduced organic carbon in the form of triose-phosphate (Triose-P). Triose-P is exported to the cytosol in strict 1:1 counterexchange with inorganic phosphate $(\mathrm{Pi})$, thus maintaining the phosphate homoeostasis of the plastid stroma. Once in the cytosol, Triose-P can be converted to Floridean Starch via UDP-Glucose (UDP-Glc) or to the soluble galactosylglycerol Floridoside via Glycerol-3-P and UDP-galactose (UDP-Gal). For clarity, the biosynthetic pathways have been simplified and abbreviated. 
The genomes of $G$. sulphuraria and C. merolae do not encode plastidic maltose or glucose transporters similar to those found in green plants ${ }^{43,88-91}$. In green plants, the glucose and the maltose transporter are involved in the export of starch breakdown products from the chloroplast to the cytosol at night ${ }^{90,91}$. Since starch is stored in the cytosol and not inside the plastid stroma in red algae, these transporters have been either lost early during the evolution of the red algae, or they have been acquired by green plants after the split of the plant linage into chlorophytes and rhodophytes.

\subsection{Concluding remarks}

Due to space constraints, this article provides only a brief survey of the most recent findings obtained in the $G$. sulphuraria Genome Sequencing Program. The preliminary analysis of the genome sequence indicates that membrane transporters are likely essential for the metabolic versatility and the stress resistance of G. sulphuraria. Functional genomics studies such as genome-wide transcript profiling and the establishment of mutants and gene transfer protocols will be required to further our understanding of the biology of this amazing organism. To better understand the metabolism of this extremophilic alga, detailed analyses of the metabolome need to be conducted under various culture conditions. For functional studies of G. sulphuraria genes and to establish reliable gene models, we will need a comprehensive collection of full-length cDNAs, representing most, if not all Galdieria genes.

\section{ACKNOWLEDGEMENTS}

We greatly appreciate technical support from Michigan State's Genomics Technology Support Facility (GTSF; http://genomics.msu.edu). This work was supported by NSF Award EF-0332882.

\section{REFERENCES}

1. Yoon, H.S., et al. (2004) A molecular timeline for the origin of photosynthetic eukaryotes. Mol. Biol. Evol. 21, 809-818

2. Yoon, H.S., et al. (2002) The single, ancient origin of chromist plastids. Proc Natl Acad Sci U S A 99, 15507-15512

3. Coppin, A., et al. (2004) Evolution of plant-like crystalline storage polysaccharide in the protozoan parasite Toxoplasma gondii argues for a red algal ancestry. J. Mol. Evol., in press

4. Ciniglia, C., et al. (2004) Hidden biodiversity of the extremophilic Cyanidiales red algae. Mol. Ecol. 13, 1827-1838

5. Albertano, P., et al. (2000) The taxonomic position of Cyanidium, Cyanidioschyzon and Galdieria: an update. Hydrobiologia 433, 137-143

6. Cozzolino, S., et al. (2000) Molecular variation in Galdieria sulphuraria (Galdieri) Merola and its bearing on taxonomy. Hydrobiologia 433, 145-151

7. Oliveira, M.C., and Bhattacharya, D. (2000) Phylogeny of the Bangiophycidae (Rhodophyta) and the secondary endosymbiotic origin of algal plastids. Am. J. Bot. 87, 482-492

8. Rothschild, L.J., and Mancinelli, R.L. (2001) Life in extreme environments. Nature 409, $1092-1101$

9. Tansey, M.R., and Brock, T.D. (1972) The upper temperature limit for eukaryotic organisms. Proc Natl Acad Sci U S A 69, 2426-2428

10. Brock, T.D. (1973) Lower pH limit for the existence of blue-green algae: evolutionary and ecological implications. Science 179, 480-483

11. Doemel, W.N., and Brock, T.D. (1970) The upper temperature limit of Cyanidium caldarium. Arch. Mikrobiol. 72, 326-332

12. Donachie, S.P., et al. (2002) Microbial community in acidic hydrothermal waters of volcanically active White Island, New Zealand. Extremophiles 6, 419-425

13. Brock, T.D. (1978) The genus Cyanidium. In Thermophilic microorganisms and life at high temperatures. (Brock, T.D., ed), 255-302, Springer-Verlag 
14. Doemel, W.N., and Brock, T.D. (1971) The physiolocial ecology of Cyanidium caldarium. J. Gen. Microbiol. 67, 17-32

15. Gross, W. (1999) Revision of comparative traits for the acido- and thermophilic red algae Cyanidium and Galdieria. In Enigmatic Microorganisms and Life in Extreme Environments (Seckbach, J., ed), 437-446, Kluwer

16. Gross, W., and Schnarrenberger, C. (1995) Heterotrophic Growth of 2 Strains of the Acido-Thermophilic Red Alga Galdieria sulphuraria. Plant Cell Physiol. 36, 633-638

17. Oesterhelt, C., et al. (1999) Characterization of a sugar/polyol uptake system in the red alga Galdieria sulphuraria. Europ. J. Phycol. 34, 271-277

18. Rigano, C., et al. (1976) Studies on utilization of 2-ketoglutarate, glutamate and other amino acids by the unicellular alga Cyanidium caldarium. Arch. Microbiol. 107, 133-138

19. Bailey, R.W., and Staehelin, L.A. (1968) The chemical composition of isolated cell walls of Cyanidium caldarium. J. Gen. Microbiol. 54, 269-276

20. Staehelin, L.A. (1968) Ultrastructural changes of the plasmalemma and the cell wall during the life cycle of Cyanidium caldarium. Proc $R$ Soc Lond B Biol Sci 171, 249-259

21. Seckbach, J., et al. (1970) Algae thrive under pure CO2. Nature 227, 744-745

22. Seckbach, J., and Libby, W.F. (1970) Vegetative life on Venus? Or investigations with algae which grow under pure CO 2 in hot acid media at elevated pressures. Space Life Sci. 2, 121-143

23. Yoshimura, E., et al. (1999) Extraordinary high aluminium tolerance of the acidophilic thermophilic alga, Cyanidium caldarium. Soil Sci Plant Nutr 45, 721-724

24. Yoshimura, E., et al. (2000) Mechanism of aluminium tolerance in Cyanidium caldarium. Hydrobiologia 433, 57-60

25. Nagasaka, S., et al. (2002) Novel iron-storage particles may play a role in aluminum tolerance of Cyanidium caldarium. Planta 215, 399-404

26. Gross, W. (2000) Ecophysiology of algae living in highly acidic environments. Hydrobiologia 433, 31-37

27. Benz, R., et al. (1980) Effects of lipid structure on the kinetics of carrier-mediated ion transport. Acta

Physiol Scand Suppl 481, 47-52

28. Benz, R., and Cros, D. (1978) Influence of sterols on ion transport through lipid bilayer membranes.

Biochim. Biophys. Acta 506, 265-280

29. Elferink, M.G.L., et al. (1992) Functional Reconstitution of Membrane Proteins in Monolayer Liposomes from Bipolar Lipids of Sulfolobus- Acidocaldarius. J. Biol. Chem. 267, 1375-1381

30. Komatsu, H., and Chong, P.L. (1998) Low permeability of liposomal membranes composed of bipolar tetraether lipids from thermoacidophilic archaebacterium Sulfolobus acidocaldarius. Biochemistry 37, 107-115

31. Jung, S., et al. (1993) Sarcina ventriculi synthesizes very long chain dicarboxylic acids in response to different forms of environmental stress. J. Biol. Chem. 268, 2828-2835

32. Burdette, D.S., et al. (2002) Physiological function of alcohol dehydrogenases and long-chain $(\mathrm{C}(30))$ fatty acids in alcohol tolerance of Thermoanaerobacter ethanolicus. Appl Environ Microbiol 68, 1914-1918

33. Jung, S., et al. (1994) A new family of very long chain alpha,omega-dicarboxylic acids is a major structural fatty acyl component of the membrane lipids of Thermoanaerobacter ethanolicus 39E. J. Lipid Res. 35, 1057-1065

34. Ford, T.W. (1979) Ribulose 1,5-bisphosphate carboxylase from the thermophilic, acidophilic alga, Cyanidium caldarium (Geitler). Purification, characterisation and thermostability of the enzyme. Biochim. Biophys. Acta 569, 239-248

35. Uemura, K., et al. (1997) Ribulose-1,5-bisphosphate carboxylase/oxygenase from thermophilic red algae with a strong specificity for CO2 fixation. Biochem Biophys Res Commun 233, 568-571

36. Okano, Y., et al. (2002) X-ray structure of Galdieria Rubisco complexed with one sulfate ion per active site. FEBS Lett. 527, 33-36

37. Gross, W., and Schnarrenberger, C. (1995) Purification and Characterization of a Galactose-1-PhosphateUDP-Glucose Uridyltransferase from the Red Alga Galdieria sulphuraria. Eur J Biochem 234, 258-263

38. Prosselkov, P.V., et al. (1996) Purification and characterization of UDP-D-galactose 4-epimerase from the red alga Galdieria sulphuraria. Physiol. Plant. 98, 753-758

39. Gross, W., et al. (2002) Characterization of a non-thermophilic strain of the red algal genus Galdieria isolated from Soos (Czech Republic). Eur. J. Phycol. 37, 477-482

40. Hardison, R.C. (2003) Comparative genomics. PLoS Biol 1, E58

41. Matsuzaki, M., et al. (2004) Genome sequence of the ultrasmall unicellular red alga Cyanidioschyzon merolae 10D. Nature 428, 653-657 
42. Weber, A.P.M., et al. (2004) EST-analysis of the thermo-acidophilic red microalga Galdieria sulphuraria reveals potential for lipid A biosynthesis and unveils the pathway of carbon export from rhodoplasts. Plant Mol.

Biol. 55, 17-32

43. Barbier, G., et al. (2005) Genome Analysis. Comparative Genomics of Two Closely Related Unicellular Thermo-Acidophilic Red Algae, Galdieria sulphuraria and Cyanidioschyzon merolae, Reveals the Molecular Basis of the Metabolic Flexibility of Galdieria and Significant Differences in Carbohydrate Metabolism of Both Algae. Plant Physiol. 137, 460-474

44. Johnson, E.M., et al. (1991) A plastome mutation affects processing of both chloroplast and nuclear DNAencoded plastid proteins. Mol. Gen. Genet. 225, 106-112

45. Thorstenson, Y.R., et al. (1998) An automated hydrodynamic process for controlled, unbiased DNA shearing. Genome Res. 8, 848-855

46. Altschul, S.F., et al. (1997) Gapped BLAST and PSI-BLAST: a new generation of protein database search programs. Nucleic Acids Res. 25, 3389-3402

47. Muravenko, O.V., et al. (2001) Chromosome numbers and nuclear DNA contents in the red microalgae Cyanidium caldarium and three Galdieria species. Eur J Phycol 36, 227-232

48. Moreira, D., et al. (1994) Characterization of 2 New Thermoacidophilic Microalgae - Genome

Organization and Comparison with Galdieria sulphuraria. FEMS Microbiol. Lett. 122, 109-114

49. Ewing, B., et al. (1998) Base-calling of automated sequencer traces using phred. I. Accuracy assessment.

Genome Res. 8, 175-185

50. Ewing, B., and Green, P. (1998) Base-calling of automated sequencer traces using phred. II. Error probabilities. Genome Res. 8, 186-194

51. Ewing, B., and Green, P. (2000) Analysis of expressed sequence tags indicates 35,000 human genes. Nat. Genet. 25, 232-234

52. Wendl, M.C., et al. (2001) Automated processing of raw DNA sequence data. IEEE Eng Med Biol Mag 20, 41-48

53. Marquardt, J., et al. (2000) Intron-exon structure and gene copy number of a gene encoding for a membrane-intrinsic light-harvesting polypeptide of the red alga Galdieria sulphuraria. Gene 255, 257-265

54. Burge, C., and Karlin, S. (1997) Prediction of complete gene structures in human genomic DNA. J. Mol. Biol. 268, 78-94

55. Lukashin, A.V., and Borodovsky, M. (1998) GeneMark.hmm: new solutions for gene finding. Nucleic Acids Res. 26, 1107-1115

56. Mathe, C., et al. (2002) Current methods of gene prediction, their strengths and weaknesses. Nucleic Acids Res. 30, 4103-4117

57. Korf, I. (2004) Gene finding in novel genomes. BMC Bioinformatics 5, 59

58. Busch, W., and Saier, M.H. (2004) The IUBMB-Endorsed Transporter Classification System. Mol.

Biotechnol. 27, 253-262

59. Busch, W., and Saier, M.H., Jr. (2002) The transporter classification (TC) system, 2002. Crit Rev Biochem Mol Biol 37, 287-337

60. Linka, N., et al. (2003) Phylogenetic relationships of non-mitochondrial nucleotide transport proteins in bacteria and eukaryotes. Gene 306, 27-35

61. Schmitz-Esser, S., et al. (2004) ATP/ADP translocases: a common feature of obligate intracellular amoebal symbionts related to Chlamydiae and Rickettsiae. J. Bacteriol. 186, 683-691

62. Reiser, J., et al. (2004) Molecular physiological analysis of the two plastidic ATP/ADP transporters from Arabidopsis. Plant Physiol. 136, 3524-3536

63. Olesen, C., et al. (2004) Dephosphorylation of the calcium pump coupled to counterion occlusion. Science $306,2251-2255$

64. Toyoshima, C., et al. (2000) Crystal structure of the calcium pump of sarcoplasmic reticulum at $2.6 \mathrm{~A}$ resolution. Nature 405, 647-655

65. De Vos, K.J., et al. (2005) Mitochondrial function and actin regulate dynamin-related protein 1-dependent mitochondrial fission. Curr. Biol. 15, 678-683

66. Kiefel, B.R., et al. (2004) Diverse eukaryotes have retained mitochondrial homologues of the bacterial division protein FtsZ. Protist 155, 105-115

67. Gilson, P.R., and Beech, P.L. (2001) Cell division protein FtsZ: running rings around bacteria, chloroplasts and mitochondria. Res Microbiol 152, 3-10 
68. Beech, P.L., et al. (2000) Mitochondrial FtsZ in a chromophyte alga. Science 287, 1276-1279

69. Ma, C., and Chang, G. (2004) Crystallography of the integral membrane protein EmrE from Escherichia coli. Acta Crystallogr. D Biol. Crystallogr. 60, 2399-2402

70. Ma, C., and Chang, G. (2004) Structure of the multidrug resistance efflux transporter EmrE from Escherichia coli. Proc Natl Acad Sci U S A 101, 2852-2857

71. Weber, A.P.M., et al. (2005) Solute transporters of the plastid envelope membrane. Annu. Rev. Plant Biol. $56,133-164$

72. Knappe, S., et al. (2003) Analysis of the plastidic phosphate translocator gene family in Arabidopsis and identification of new phosphate translocator-homologous transporters, classified by their putative substrate-binding site. Plant Physiol. 131, 1178-1190

73. Niewiadomski, P., et al. (2005) The Arabidopsis Plastidic Glucose 6-Phosphate/Phosphate Translocator GPT1 Is Essential for Pollen Maturation and Embryo Sac Development. Plant Cell 17, 760-775

74. Knappe, S., et al. (2003) Characterization of two functional phosphoenolpyruvate/phosphate translocator (PPT) genes in Arabidopsis--AtPPT1 may be involved in the provision of signals for correct mesophyll development. Plant J. 36, 411-420

75. Eicks, M., et al. (2002) The plastidic pentose phosphate translocator represents a link between the cytosolic and the plastidic pentose phosphate pathways in plants. Plant Physiol. 128, 512-522

76. Kammerer, B., et al. (1998) Molecular Characterization of a Carbon Transporter in Plastids from Heterotrophic Tissues: The Glucose6-Phosphate/Phosphate Antiporter. Plant Cell 10, 105-117

77. Fischer, K., et al. (1997) A new class of plastidic phosphate translocators: A putative link between primary and secondary metabolism by the phosphoenolpyruvate/phosphate antiporter. Plant Cell 9, 453-462

78. Loddenkötter, B., et al. (1993) Expression of the functional mature chloroplast triose phosphate translocator in yeast internal membranes and purification of the histidine-tagged protein by a single metal-affinity chromatography step. Proc. Natl. Acad. Sci. USA 90, 2155-2159

79. Renné, P., et al. (2003) The Arabidopsis mutant dct is deficient in the plastidic glutamate/malate translocator DiT2. Plant J. 35, 316-331

80. Weber, A., et al. (1995) The 2-oxoglutarate/malate translocator of chloroplast envelope membranes:

Molecular cloning of a transporter containing a 12-helix motif and expression of the functional protein in yeast cells. Biochemistry 34, 2621-2627

81. Flügge, U.I. (1999) Phosphate Translocators in Plastids. Annu. Rev. Plant Physiol. Plant Mol. Biol. 50, 27-

45

82. Flügge, U.I., and Heldt, H.W. (1986) Chloroplast phosphate-triose phosphate-phophoglycerate translocator: Its identification, isolation, and reconstitution. In Methods of Enzymology Vol. 125, 716-730, Academic Press Inc.

83. Flügge, U.I., et al. (1991) The Major Chloroplast Envelope Polypeptide Is the Phosphate Translocator and Not the Protein Import Receptor. Nature 353, 364-367

84. Flügge, U.I. (1992) Reaction mechanism and asymmetric orientation of the reconstituted chloroplast phosphate translocator. Biochim. Biophys. Acta 1110, 112-118

85. Fliege, R., et al. (1978) Specific transport of inorganic phosphate, 3-phosphoglycerate and triosephosphates across the inner membrane of the envelope in spinach chloroplasts. Biochim. Biophys. Acta 502, 232-247

86. Flügge, U.I., et al. (1989) The triose phosphate-3-phosphoglycerate-phosphate translocator from spinach chloroplasts: nucleotide sequence of a full-length cDNA clone and import of the in vitro synthesized precursor protein into chloroplasts. EMBO J. 8, 39-46

87. Flügge, U.I. (1999) Phosphate translocators in plastids. Annu. Rev. Plant Physiol. Plant Mol. Biol. $50,27-$

45

88. Niittyla, T., et al. (2004) A previously unknown maltose transporter essential for starch degradation in leaves. Science 303, 87-89

89. Weber, A., et al. (2000) Identification, Purification, and Molecular Cloning of a Putative Plastidic Glucose Translocator. Plant Cell 12, 787-801

90. Weise, S.E., et al. (2004) Maltose is the major form of carbon exported from the chloroplast at night. Planta 218, 474-482

91. Weber, A.P.M. (2004) Solute transporters as connecting elements between cytosol and plastid stroma. Curr. Opin. Plant Biol. 7, 247-253 